\title{
Study on the Volatility Relationship between the Price of China's Live-hogs Industrial Chain and CPI
}

\author{
Xuanmin Zhang \\ School of Economics, Shanghai University Shanghai, China \\ 772320152@qq.com
}

\begin{abstract}
This paper uses the SAVR model to study the dynamic relationship between monthly corn prices, live hog prices, pork prices, and CPI volatility from January 2011 to August 2021. It is found that: 1. live-hog prices is the cause of pork price fluctuation, and live hogs and pork prices is the cause of $\mathrm{CPI}$ change. 2. live hog prices has short-term positive brunt on $\mathrm{CPI}$, and pork prices has short-term positive and negative impact on CPI. 3. pork prices change is mainly caused by live hog price and its own change, and CPI change is mainly caused by spontaneous factors, live hog prices and pork prices change. Based on the above research, relevant policy recommendations to smooth out the fluctuation of pork prices are proposed.
\end{abstract}

Keywords: Live Hogs Chain; Pork Prices; CPI; SVAR Model.

\section{Introduction}

Pork is the main meat food for China's residents and also an important component of the consumer price index (CPI), whose price fluctuations not only affect the upstream and downstream enterprises of the live hogs industry chain, but also spread to the lives of urban and rural residents as well as the operation of the national macroeconomic. It is of great practical significance to study the impact of pork prices, live-hog industry chain prices and pork prices fluctuations on CPI.

\section{Review of the Literature}

Domestic research on the price fluctuations of the live hogs industry chain and the relationship with CPI changes is mainly in two areas: (1) research on price fluctuations of the live hogs industry chain. Liu Qingquan, Zhou Faming et al. (2012) [1], using co-integration theory and SVAR model analysis, concluded that shocks in the price of Live-hogs themselves and piglets have an important impact on the price fluctuations of live hogs, and cause fluctuations in the price of pork. Zhou Cong and Wang, Xinhua (2015) [2] used the VAR model to find that the degree of influence of corn prices on live hog prices is greater than soybean prices, and the prices of corn and soybeans are more influenced by live hog prices. Yu Xutao, Fang Yongmei (2019) [3] with the help of VAR model, the study found that in addition to the price law of pork itself, the prices of soybean meal and live hogs contribute more to the price of pork, and the shocks of corn, piglets and fattening hog with feed have no greater impact on the price of pork in the short and long term. (2) Research on the relationship between pork prices and CPI. Based on 2006-2020 data, Yi Zhenhua (2020)[4] found that the high weight of hog prices in the CPI basket, the high volatility of hog prices, and the strong transmission effect of hog prices on the prices of substitutes are the main reasons for the high consistency of hog prices and CPI trends. Using a VAR model, Xing Linjing, Guo Xuan et al. (2021)[5] showed that there is a one-way Granger causality between pork prices and CPI, and the impact of pork price changes on CPI is more significant within 2 years.

Throughout the above studies, there is no direct research involving price and CPI fluctuations in the upstream and downstream hog chains, while most of the studies are based on the VAR (vector auto-regressive) model, which has research flaws as this model does not consider the current period effects. The SVAR (structural vector auto-regressive) model used in this paper allows for the existence of current period relationships among the variables, captures the immediate structural relationships among the variables, and can calculate the orthogonality impulse functions to separately 
analyze the impact of shocks on the other variables [6], which is an improvement of the VAR model and makes the research analysis more complete.

\section{Data and Models}

\subsection{Selection and Sources of Data}

In order to make the sample more representative and to be able to explore the long-term movement relationship of the four. This paper selects monthly data from January 2011 to August 2021, using ymp for corn price (yuan/kg), szp for hog price (yuan/kg), zrp for pork price (yuan/kg), and CPI for consumer price index (same month of the previous year $=100$ ). Corn, hog and pork prices are sourced from the Iimedia Data Center (https://data.iimedia.cn/) and the consumer price index for residents from the National Bureau of Statistics (http://www.stats.gov.cn/). In order to eliminate the seasonal effect of the data and the possible heteroskedasticity problem in the modeling process, GensusX12 seasonal adjustment and logarithmic processing were performed in the study using Eviews software, respectively. The lnymp, lnszp, lnzrp, and lncpi denote the logged maize, hog, and pork prices and the residential consumer price index, respectively.

\subsection{Basis Test for SVAR Model}

First, the model data were tested for smoothness, and the test results showed that the original data were all non-smooth series, and the absolute values of the ADF statistics of the data after passing the first-order difference (the difference data were expressed as dlnymp, dlnszp, dlnzrp, and dlncpi tables) were all above the $1 \%$ critical value, rejecting the original hypothesis and indicating that the series were all smooth series. Secondly, the optimal lag order was determined by using the AIC value, SC value and HQ value to determine the optimal lag order of the SVAR model to be 2nd order. Again, the smoothness test of the SVAR model, there are four endogenous variables with lag length 2 in this paper, there are eight characteristic roots, and the mode of the inverse of the characteristic roots are all less than 1, indicating that the SVAR model is stable. Finally, the Granger causality Test, the test results show that at 5\% confidence level, the change in hog price and pork price is the cause of the change in CPI, and the change in hog price is the cause of the change in pork price.

\subsection{Structural Design of the SVAR Model}

The general expression for the SVAR model is

$$
c_{0} y_{t}=\Gamma_{1} y_{t-1}+\Gamma_{2} y_{t-2}+\cdots+\Gamma_{p} y_{t-p}+\mu_{t}
$$

The above equation describes the SVAR(p) model containing delayed orders $\mathrm{p}$ and containing $\mathrm{k}$ variables, among,

$$
\begin{aligned}
c_{0} & =\left(\begin{array}{cccc}
1 & c_{12} & \cdots & c_{1 k} \\
c_{21} & 1 & \cdots & c_{2 k} \\
\vdots & \vdots & 1 & \vdots \\
c_{k 1} & c_{k 2} & \cdots & 1
\end{array}\right) \\
\Gamma_{i} & =\left(\begin{array}{cccc}
r_{11}^{i} & r_{12}^{i} & \cdots & r_{i k}^{i} \\
r_{21}^{i} & r_{22}^{i} & \cdots & r_{2 k}^{i} \\
\vdots & \vdots & \ddots & \vdots \\
r_{k 1}^{i} & r_{k 2}^{i} & \cdots & r_{k k}^{i}
\end{array}\right), \mathrm{i}=1,2, \ldots, \mathrm{p}
\end{aligned}
$$




$$
\mu_{t}=\left(\begin{array}{c}
\mu_{i t} \\
\mu_{2 t} \\
\vdots \\
\mu_{k t}
\end{array}\right)
$$

According to the SVAR model identification condition, the k-dimensional p-order model needs to impose $\mathrm{k}(\mathrm{k}-1) / 2$ constraints on the structural equation to identify the structural shock. This paper is a 4-dimensional 2nd-order SVAR model, so there are 6 constraints. Also in this paper, assuming that the SVAR model is of type $A B$, so have $A \varepsilon_{t}=B \mu_{t}, A=c_{0}$, and the order of endogenous variables $\operatorname{arey}_{\mathrm{t}=}(\mathrm{dlnymp}$, dlnszp, dlnzrp, dlncpi). According to the Cholesky constraint and related economic theory, the specific constraints are as follows: corn price does not respond to the current CPI, current pork price, and current hog price changes, that $c_{14}=0, c_{13}=0, c_{12}=0$; hog prices do not respond to current $\mathrm{CPI}$, current pork price changes, that $c_{24}=0, c_{23}=0$; pork prices do not respond to current CPI changes, that $c_{34}=0$. Therefore, this paper imposes the following constraints on matrices $\mathrm{A}$ and $\mathrm{B}$, respectively.

$$
\begin{aligned}
A & =\left(\begin{array}{cccc}
1 & 0 & 0 & 0 \\
c_{21} & 1 & 0 & 0 \\
c_{31} & c_{32} & 1 & 0 \\
c_{41} & c_{42} & c_{43} & 1
\end{array}\right) \\
B & =\left(\begin{array}{cccc}
b_{11} & 0 & 0 & 0 \\
0 & b_{22} & 0 & 0 \\
0 & 0 & b_{33} & 0 \\
0 & 0 & 0 & b_{44}
\end{array}\right)
\end{aligned}
$$

\section{Empirical Analysis}

\subsection{Impulse Response Function Analysis}

Results of the main impulse responses in the live hog chain .It can be seen in Fig. 1 that live hog prices has a longer-term positive impact on maize price, with the impact gradually increasing in the 2nd-3rd period, reaching the maximum in the 3rd period, then gradually decaying, and gradually converging to zero in the 6th period. Live hogs are a downstream product of corn, when the price of live hogs rises, it will gradually drive upstream corn prices, but its impact will gradually weaken over time; pork prices have a short-term negative impact on live hog prices, with the largest impact in period 2 , then gradually decaying, and gradually tending to zero after period 4 . Generally speaking, the shortterm pork price increase will lead to the accelerated slaughter of live hogs, resulting in a fall in the price of pigs, but the fall is not sustained, and will subsequently rise with the rise in pork prices; hog prices have a longer-term positive impact on pork prices, with rapid decay from period 1-3, decaying to a lesser extent from period 3-6, and gradually tending to 0 after period 6 . This indicates the positive impact of live hog prices on pork prices, and its impact cycle lasts is about half a year.

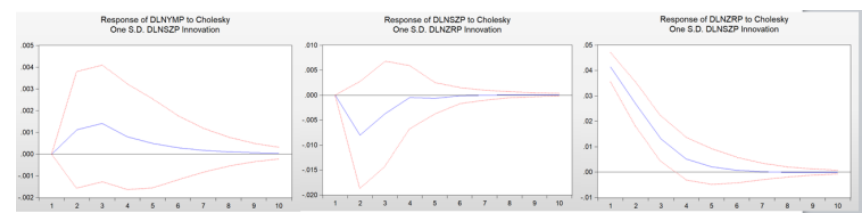

Fig 1. Results of the main impulse responses of the live hog chain 
Results of Pork Prices and CPI Impulse Response. It is manifest in Fig. 2 that CPI has a longerterm negative shock to pork prices, the shock gradually increases in the 2 nd-3rd period and reaches the maximum in the 3rd period, the shock gradually weakens after the 3rd period, and by degrees tends to 0 value after the 6 th period. It means that the rise of CPI will suppress the rise of pork price, probably because the rise of pork price leads to the rise of price. The state introduces relevant policies to increase the slaughter of live hogs and suppress the rise of pork price by control the rise of price. Live hog prices has a short-term positive shock to CPI, the positive shock decreases rapidly in the 1st-3rd period, and stabilizes near the 0 value after the 4th period. It indicates that short-term live hog price will lead to the rise of CPI, but the impact time is short; pork price has short-term positive and negative shocks to CPI, with positive shocks to CPI in period 1, negative shocks in period 2, positive shocks in period 3 , and the magnitude of shocks diminishes, and stabilizes near the value of 0 after period 4 . It shows that the rise in pork prices leads to a rise in CPI in the current period, yet the impact is not sustained, and a negative shock may be generated in period 2 because of the high rise in CPI in the previous period and the lack of upward momentum in the current period, and a positive shock resumes in period 3 , but the impact is gradually weakened.

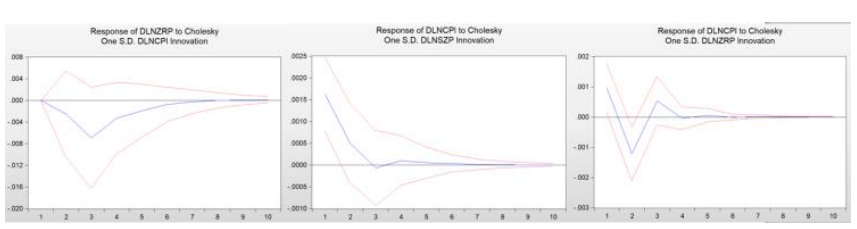

Fig 2. Model for Pork prices and CPI impulse response

\subsection{Variance Decomposition}

From TABLE I, it can be show that the variance of corn price change is mainly caused by its own; The variance (about $92.50 \%$ ) of live hog price change is caused by its own and by corn price change (about $4.40 \%$ ); the variance (about $85.37 \%$ ) of pork price change is due to live hog price change, about $8.25 \%$ by its own price change and about $4.62 \%$ by corn price change. About $77.13 \%$ of the variance analysis of CPI changes are explained by its own changes, about $10.87 \%$ by changes in hog prices, about $9.27 \%$ by changes in pork prices and about $2.73 \%$ by changes in corn prices.

Table 1. Results of variance decomposition (mean of 10th order)

\begin{tabular}{|c|c|c|c|c|}
\hline & \multicolumn{4}{|c|}{ Variance decomposition } \\
\cline { 2 - 5 } & Corn prices & Live hog prices & Pork prices & CPI \\
\hline Corn prices & $98.14 \%$ & $4.40 \%$ & $4.62 \%$ & $2.73 \%$ \\
\hline Live hog prices & $1.14 \%$ & $92.50 \%$ & $85.37 \%$ & $10.87 \%$ \\
\hline Pork prices & $0.29 \%$ & $1.47 \%$ & $8.25 \%$ & $9.27 \%$ \\
\hline CPI & $0.43 \%$ & $1.63 \%$ & $1.75 \%$ & $77.13 \%$ \\
\hline
\end{tabular}

\section{Conclusions and Recommendations}

Through the above study, we found that: live hog prices is caused to the Granger of pork price, live hog and pork price is the Granger cause of CPI. In the impulse response analysis of live hog industry chain, live hog price has a longer-term positive shock to corn price, pork price has a shortterm negative shock to live hog price, and live hog price has a longer-term positive shock to pork price. In the impulse response analysis of pork prices and CPI, CPI has a longer-term negative shock to pork prices, live hog prices have a short-term positive shock to CPI, and pork prices have a shortterm positive and negative shock to CPI. In the impulse response analysis of pork price and CPI, CPI has a longer-term negative impact on pork price, live hog prices has a short-term positive impact on CPI, and pork price has a short-term positive and negative impact on CPI; changes in corn and live hog price are mainly caused by their own changes, changes in pork price are mainly caused by hog 
price and their own changes, and the variance of CPI changes is mainly caused by their own, live hog price and pork price changes. Based on the above findings, the following policy recommendations are made:

On one hand: Strengthening policy regulation of the whole live hog industry chain. Firstly, establish an early warning mechanism for information on the whole live hog industry chain, regularly announce the prices of corn, wheat, breeding hogs, fattening hogs and pork, etc. involving the upstream and downstream industry chains on a monthly and quarterly basis, and establish a dynamic prediction model to issue corresponding policy guidelines for sudden price changes; Secondly, guide and give full play to the government's macro-control mechanism on corn, wheat and other planting industries to calm food price fluctuations, while strengthening the policy guidance and regulation of small and medium-sized retail pig farming and large-scale slaughtering enterprises, making full use of financial subsidies, window guidance and storage policies to stabilize market supply and stabilize fluctuations in live hog prices; finally, develop fully to the regulatory and supervisory role of grassroots animal husbandry authorities, combining market conditions and reasonably guiding farmers to add and reduce capacity and other work in order to stabilize the supply of pork.

One the other hand: Improving the level of large-scale pig farming. China's live hog farming industry has long been dominated by free-range farming, and the concentration of the industry is low, with a large number of free-range farmers and small and medium-sized farms. According to China Animal Husbandry and Veterinary Yearbook, in 2017, there were 407 farmers with more than 50,000 hogs per year, accounting for only $0.0011 \%$ of the total number of farmers. In 2020, the top ten hog breeding enterprises in China slaughtered 64,536,500 hogs, with a market share of only $12.25 \%$. The low concentration of China's live hog farming industry and the inherent growth cycle of pigs jointly determine the cyclical fluctuations of live hog market prices. The expansion of live hog farming scale can not only reduce the cost and improve the ability of epidemic prevention and control, but also increase the concentration of the market, which is convenient for policy regulation and control and suppress the fluctuation of hog price.

Third: Strengthening the use of financial instruments such as insurance and futures. Animal disease is the main risk faced by the animal husbandry industry. Live hog insurance is an important protection in the hog breeding chain, which should be explored to strengthen the claim amount and claim efficiency of insurance for breeding sows and fattening hogs, while a special epidemic disaster insurance can be considered for major epidemics to stabilize live hog production. Hog futures is a futures contract with live hogs as the underlying, which is ready to be listed and traded on Dalian Commodity Exchange in January 2021 after nearly 20 years of brewing. The reasonable use of hog futures can not only provide risk hedging tools for industry chain customers, but also guide the fluctuation of hog spot prices and give reasonable expectations to the market.

\section{References}

[1] Liu QQ, Zhou FM, Li Y. Price relationships and dynamic effects of the live hog industry chain in China [J]. Statistics and Decision Making, 2012(2):131.

[2] Zhou C, Wang XH. The interaction of live hog price, corn price and soybean price based on VAR model [J]. Jiangsu Agricultural Science, 2015,43(6):464.

[3] Yu XT, Fang YM. Analysis of pork price volatility based on VAR model[J]. Shanxi Science and Technology, 2019,34(2):1-5.

[4] Yi ZH. Pork price change characteristics, trend outlook and impact on CPI--analysis based on the perspective of hog cycle [J]. Zhejiang Finance, 2020(10):32.

[5] Yin LY, Guo X, Hu J. Research on the correlation between pork prices and CPI based on VAR model in China [J]. Rural Economy and Technology, 2021, 32(13):64.

[6] Jia YN, Gao QQ. Monetary policy, credit orientation and financial support to stabilize enterprises and preserve employment - an empirical study based on SVAR model[J]. North China Finance, 2021(9):38. 